\title{
Critical appraisal of the role of sorafenib in the management of hepatocellular carcinoma
}

This article was published in the following <ppname> journal:

Hepatic Medicine: Evidence and Research

I November 2010

Number of times this article has been viewed

Jen-Jung $\operatorname{Pan}^{\prime}$

Milind Javle ${ }^{2}$

Mie Mie Thinn ${ }^{3}$

Chung-Tzu Hsueh ${ }^{4}$

Chung-Tsen Hsueh ${ }^{5}$

'Division of Gastroenterology, Hepatology and Nutrition, Department of Internal Medicine,

The University of Texas Medical School at Houston, Houston, TX,

USA; ${ }^{2}$ Department of Gastrointestinal Medical Oncology, The University of Texas MD Anderson Cancer Center, Houston, TX, USA; ${ }^{3}$ Division of Oncology, University of Southern California, Keck School of Medicine, Los Angeles, CA, USA; ${ }^{4}$ Dental

Department, Cathay General Hospital, Taipei, Taiwan; ${ }^{5}$ Division of Medical Oncology and Hematology, Loma Linda University Medical Center, Loma Linda, CA, USA
Correspondence: Chung-Tsen Hsueh Division of Medical Oncology and Hematology, Loma Linda University Medical Center, I I I75 Campus, CSP I 1015 , Loma Linda, CA 92354, USA

$\mathrm{Tel}+\mathrm{I} 9095588107$

Fax + I 9095580219

Email chsueh@llu.edu

\begin{abstract}
Sorafenib is an oral multiple kinase inhibitor that blocks Raf, vascular endothelial growth factor receptor, and platelet-derived growth factor receptor. It has been approved in the US and Europe for the treatment of advanced hepatocellular carcinoma (HCC). Sorafenib has demonstrated a 44\% increase in survival for advanced HCC patients, compared with best supportive care alone. We have reviewed the pharmacology, pivotal studies, and safety data for this agent. Sorafenib is the first systemic drug demonstrating a significant survival benefit, and is the standard of care for patients with advanced HCC for whom no potential curative option is available.
\end{abstract}

Keywords: sorafenib, hepatocellular carcinoma, multiple kinase inhibitor

\section{Introduction}

Hepatocellular carcinoma (HCC) has become a global health problem, with more than half a million new cases diagnosed worldwide each year. ${ }^{1} \mathrm{HCC}$ is associated with a high mortality rate, and is the leading cause of liver-related death in patients with compensated cirrhosis. ${ }^{2}$ The incidence of $\mathrm{HCC}$ is increasing in several developed countries, including the US. Between 1995 and 1998 alone, there was a 25\% increase of HCC incidence in the US. ${ }^{3}$ It has been estimated that the number of cases of HCC will continue to increase by $81 \%$ to the year 2020 , predominantly due to hepatitis $\mathrm{C}$ (HCV) infection. ${ }^{4}$ Nevertheless, the incidence of HCC is decreasing in some developing countries as a result of the implementation of universal hepatitis B vaccination. ${ }^{5}$

Older age ( $>50$ years), male gender, severity of compensated liver cirrhosis at presentation, and sustained activity of liver disease are important predictors of HCC, independent of etiology of cirrhosis. ${ }^{6}$ Males typically have a two- to four-fold higher risk of developing $\mathrm{HCC}$ than females. Age may serve as a surrogate for the duration of underlying liver disease. More than $80 \%$ of patients with HCC have cirrhosis, which remains the major risk factor. Therefore, any cause of liver cirrhosis is associated with increased risks of HCC. HCV infection, hepatitis B (HBV) infection, and heavy alcohol use remain the most common causes of cirrhosis in Western countries.

Once the diagnosis of $\mathrm{HCC}$ is made and confirmed, there are several treatment options depending on the size and number of tumors, underlying liver function, presence or absence of tumor vascular invasion, and the patient's performance status. Hepatic resection may be an option for those with $\mathrm{HCC}$ in a noncirrhotic liver or those with HCC and cirrhosis but without portal hypertension and with normal bilirubin. Portal hypertension is defined as the presence of hepatic venous pressure gradient 
greater than $10 \mathrm{mmHg}$, esophageal varices, or splenomegaly with a platelet count less than $100,000 / \mathrm{mm}^{3}$. Subjects without relevant portal hypertension and normal bilirubin can achieve a $70 \%$ five-year survival after hepatic resection, whereas survival is only $50 \%$ in those with portal hypertension, and even lower with both adverse factors. ${ }^{7}$ Hepatic resection is associated with a high tumor recurrence rate, ie, up to $70 \%$ in five years, either from true recurrence or development of de novo tumors.

Liver transplantation is the mainstay treatment for those with HCC and cirrhosis. During transplantation, not only the tumor itself but also the cirrhotic liver that provides the background for new tumor development will be removed. For those who meet the Milan criteria (solitary HCC less than $5 \mathrm{~cm}$ in size or up to three tumors with each tumor less than $3 \mathrm{~cm}$ in size), transplantation provides a five-year survival exceeding $70 \%$ while maintaining a low recurrence rate of less than $15 \% .{ }^{7,8}$ In spite of being the best option for patients with HCC, transplantation is limited by the current shortage of available donors. To avoid dropout while awaiting transplantation, transplant candidates usually receive locoregional treatments, such as transarterial chemoembolization or percutaneous ablation, especially if the waiting time exceeds six months. ${ }^{9}$ Due to the small risk of tumor seeding in the needle tract associated with percutaneous ablation, transarterial chemoembolization is more favored as a bridging therapy for transplant candidates.

For those with small unresectable HCC, percutaneous ablation, such as percutaneous ethanol injection and radiofrequency ablation, offers comparable survival with that of resection. ${ }^{10-13}$ In addition to similar survival compared with surgery, percutaneous ablation therapy is relatively simple, low-cost, repeatable, and safe. Compared with percutaneous ethanol injection, radiofrequency ablation has a higher rate of complete tumor necrosis (radiofrequency ablation 90\% versus percutaneous ethanol injection $80 \%$ ), requires fewer treatment sessions (radiofrequency ablation 1.2 versus percutaneous ethanol injection 4.8), and is associated with better local recurrence-free survival compared with percutaneous ethanol injection. Nevertheless, the incidence of adverse events is not significantly different between these two treatment modalities.

In addition to being used as a bridging therapy before transplantation, transarterial chemoembolization also serves as a palliative measure for those with unresectable HCC and who are not amenable to percutaneous ablation. In a systematic review of randomized, controlled trials involving various treatment modalities in patients with unresectable HCC, arterial embolization improved two-year survival compared with controls (odds ratio [OR] $0.53 ; 95 \%$ confidence interval [CI], 0.32-0.89; $P=0.017) .{ }^{14}$ Sensitivity analysis showed a significant benefit of transarterial chemoembolization (OR 0.42 ; $95 \%$ CI $0.20-0.88$ ) but none with bland embolization alone (OR 0.59; 95\% CI 0.29-1.20). Radioembolization with microspheres carrying yttrium 90 injected via the intrahepatic arterial route has been proposed as an alternative approach to transarterial chemoembolization. ${ }^{15}$ This method can deliver radiation to the HCC-associated capillary bed. Radioembolization requires only single-dose administration, and has been shown to be therapeutically equivalent to transarterial chemoembolization. ${ }^{16}$

Patients with advanced HCC who are not candidates for local approaches are considered for systemic therapy. Systemic therapy with cytotoxic agents, such as doxorubicin, provides marginal benefits without improvement in overall survival. ${ }^{17}$ Investigation in hepatocarcinogenesis has led to the discovery of key molecular targets in HCC, such as angiogenesis, epidermal growth factor receptor, and mammalian target of rapamycin. A growing number of molecularly targeted therapies for HCC are currently at different stages of clinical development. ${ }^{18}$ Sorafenib (Nexavir ${ }^{\circledR}$, Bayer Healthcare AG, Leverkusen, Germany), a multikinase inhibitor, is the only such drug that has been approved in the US and Europe for treatment of unresectable HCC. In this review, we provide a critical appraisal of sorafenib in the management of HCC.

\section{Mechanism of action and pharmacologic effects}

Sorafenib inhibits multiple tyrosine and serine/threonine kinases involved in cell proliferation and angiogenesis. These kinases include Raf kinase, vascular endothelial growth factor receptor (VEGFR) kinases, platelet-derived growth factor receptor (PDGFR) kinase, c-kit receptor kinase, RET receptor kinase, and FMS-like tyrosine kinase $3 .{ }^{19,20}$ Disruption of PDGFR and VEGFR signaling pathways inhibits angiogenesis. Raf is an essential serine/threonine kinase in the mitogen-activated protein (MAP) kinase signaling pathway, and is a downstream effector of Ras, which is activated in many human malignancies. ${ }^{21}$ When the Raf-MAP kinase pathway is activated, extracellular signal-regulated kinase (ERK) will become phosphorylated. Subsequently, phosphorylated ERK (pERK) can be translocated to the nucleus where it can regulate gene expression by modulating various transcription factors and target genes. Preclinical models have demonstrated that HCC exhibits activation of 
MAP signaling and overexpression of angiogenesis factors. Sorafenib blocks the Raf-MAP kinase and ERK pathway, inhibits tumor angiogenesis, and induces tumor cell apoptosis in HCC cell lines. ${ }^{22}$

Pharmacodynamic assay for Raf-MAP kinase inhibition by sorafenib has been investigated in patients' peripheral blood lymphocytes collected at different time points in a Phase I study. ${ }^{23}$ Lymphocytes were activated with phorbol myristate acetate, and flow cytometric analyses of pERK were performed. The phorbol myristate acetate-induced pERK in peripheral blood lymphocytes from six patients was almost completely eradicated by sorafenib after 21 days of the maximally tolerated dose. In a Phase II study of sorafenib in advanced HCC, immunohistochemical study in pretreatment tumor biopsies using an antibody against pERK was performed in 33 patients (of 137 enrolled patients), and pERK staining was more intense in the nuclei of tumor tissues. ${ }^{24}$ There was a significant difference in time to progression between patients with higher tumor cell pERK staining intensity versus those with lower intensity. Patients with tumors exhibiting higher $\mathrm{pERK}$ staining intensity had a longer time to progression. A similar finding was also noted in a retrospective biomarker analysis of the Phase III Sorafenib HCC Assessment Randomized Protocol (SHARP) trial. ${ }^{25}$ The biomarker study from the SHARP trial has further revealed that low hepatocyte growth factor and high c-kit serum levels at baseline were associated with improved survival in multivariate analysis. These findings are exploratory and require validation in perspective studies.

\section{Pharmacokinetics}

The recommended dose of sorafenib is $400 \mathrm{mg}$ orally twice a day, and should be administered to patients who are fasting or with a moderate-fat meal. A high-fat meal reduces sorafenib's bioavailability by about $30 \%$. The plasma protein binding of sorafenib is about $99 \%$ in patients with normal renal and hepatic function. ${ }^{26}$ Sorafenib is primarily metabolized in the liver, predominantly via Phase I oxidation by cytochrome $\mathrm{P} 450$ (CYP) 3A4, and Phase II conjugation by uridine 5'-diphosphoglucuronosyltransferase..$^{27,28}$ Approximately 50\% of orally administered sorafenib is recovered as unchanged drug in the feces, due to either biliary excretion or lack of absorption. Urinary excretion accounts for approximately $20 \%$ of drug metabolism. The mean terminal elimination half-life of sorafenib is approximately $24-38$ hours, and sorafenib exposures reach steady state within seven days of dosing. ${ }^{29}$

Because sorafenib is mainly metabolized by CYP3A4, its pharmacokinetics may be affected by drugs modulating the function of CYP3A4. Concurrent administration of rifampin, a CYP3A4 inducer, has been shown to decrease the area under the plasma concentration versus time curve (AUC) of sorafenib by $37 \%$. Other CYP3A4 inducers, including carbamazepine, phenobarbital, and dexamethasone, may decrease the AUC of sorafenib. ${ }^{30}$ Neomycin decreases the AUC of sorafenib by $54 \%$, probably due to eradication of gastrointestinal bacteria and therefore preventing the enterohepatic recycling of sorafenib. ${ }^{26}$ Due to potential drug interaction, patients taking medications such as warfarin, digoxin, and quinidine may require close monitoring during sorafenib treatment. ${ }^{26,31}$

It has been shown that the AUC for sorafenib is $30 \%-45 \%$ lower in Japanese or Chinese than in Caucasian populations, and the clinical significance of this finding remains unknown. ${ }^{30,32}$ The Child-Pugh scoring system uses clinical and laboratory information to stratify disease severity, surgical risk, and overall prognosis in patients with liver cirrhosis. ${ }^{33}$ The AUC of sorafenib in patients with mild (Child-Pugh A) and moderate (Child-Pugh B) hepatic impairment has been shown to be $23 \%-65 \%$ lower than in subjects with normal hepatic function, and there are no significant differences in sorafenib pharmacokinetics between patients with Child-Pugh A and Child-Pugh B hepatic impairment. ${ }^{24,34}$ No significant difference in sorafenib pharmacokinetics has been shown between patients with normal renal function and mild-to-moderate renal insufficiency. ${ }^{30,35}$ The pharmacokinetics of sorafenib has not been studied in patients with severe hepatic impairment (Child-Pugh C) or severe renal insufficiency (creatinine clearance less than $30 \mathrm{~mL} / \mathrm{min}$ ). In a Phase I pharmacokinetic study of sorafenib in patients with hepatic or renal dysfunction, Miller et al showed that patients with severe renal or hepatic dysfunction frequently developed intolerance to sorafenib. ${ }^{35}$ Therefore, it is recommended to start at a lower dose in these patient populations, followed by close monitoring and gradual dose escalation.

\section{Pivotal studies of sorafenib in HCC}

Two Phase III randomized studies, ie, SHARP and the Asia-Pacific study, comparing sorafenib with placebo as first-line systemic treatment in patients with advanced HCC, have demonstrated the efficacy of sorafenib. ${ }^{36,37}$ Patient characteristics and outcome measures of these two studies are summarized in Tables 1 and 2.

The SHARP study was designed for the regulatory approval of sorafenib in the US and Europe, and was a randomized, double-blinded, placebo-controlled, Phase III study conducted in Europe, the US, and Australasia. It enrolled 
Table I Patient characteristics in SHARP and Asia-Pacific studies

\begin{tabular}{|c|c|c|c|c|}
\hline \multirow[t]{2}{*}{ Characteristics } & \multicolumn{2}{|l|}{ SHARP } & \multicolumn{2}{|c|}{ Asia-Pacific } \\
\hline & $\begin{array}{l}\text { Sorafenib } \\
(n=299)\end{array}$ & $\begin{array}{l}\text { Placebo } \\
(n=303)\end{array}$ & $\begin{array}{l}\text { Sorafenib } \\
(n=I 50)\end{array}$ & $\begin{array}{l}\text { Placebo } \\
(n=76)\end{array}$ \\
\hline Median age (years) & 64.9 & 66.3 & 51 & 52 \\
\hline Male (\%) & 87 & 87 & 85 & 87 \\
\hline Europe/America/Asia; (\%) & $88 / 12 / 0$ & $87 / 13 / 0$ & $0 / 0 / 100$ & $0 / 0 / 100$ \\
\hline Hepatitis B/C status (\%) & $19 / 29$ & $18 / 27$ & $70.7 / 10.7$ & $77.6 / 3.9$ \\
\hline ECOG performance status 0,1 , or $2(\%)$ & $54 / 38 / 8$ & $54 / 39 / 7$ & $26 / 69 / 5$ & $28 / 67 / 5$ \\
\hline BCLC Stage C (\%) & 82 & 83 & 95 & 96 \\
\hline Child-Pugh Class A/B (\%) & $95 / 5$ & $98 / 2$ & $97 / 3$ & $97 / 3$ \\
\hline Macroscopic vascular invasion (\%) & 36 & 41 & 36 & 34 \\
\hline Extrahepatic spread (\%) & 53 & 50 & 69 & 68 \\
\hline
\end{tabular}

Abbreviations: ECOG, Eastern Cooperative Oncology Group; BCLC, Barcelona Clinic Liver Center.

602 patients with advanced HCC, well-preserved liver function, and no prior systemic therapy. Patients were randomized in a 1:1 ratio to receive either oral sorafenib (400 $\mathrm{mg}$ twice daily) or placebo. Primary endpoints were overall survival and time to symptomatic progression. Secondary outcomes included the time to radiologic progression and safety. The primary outcomes were evaluated based on the intention-totreat principle. The number of patients needed for this study was calculated on the basis of overall survival, with a power of $90 \%$ to detect a $40 \%$ improvement in overall survival in the sorafenib group.

In the SHARP study, patients were well balanced with respect to baseline characteristics (Table 1). As summarized in Table 2, this study demonstrated a significant improvement in median overall survival on sorafenib versus placebo (10.7 versus 7.9 months; hazard ratio [HR] in the sorafenib group $0.69 ; 95 \%$ CI $0.55-0.87 ; P<0.001)$. There was significant prolongation of median time to radiologic progression in the sorafenib group $(5.5$ versus 2.8 months; $P<0.001)$. In the sorafenib group, seven patients $(2.3 \%)$ had a partial response and $71(71 \%)$ had stable disease (according to the Response Evaluation Criteria in Solid Tumors [RECIST]), whereas in the placebo group, two patients (1\%) had a partial response and 204 patients (67\%) had stable disease. There were no complete responses in either group. The disease-control rate, defined as complete/partial response or stable disease by RECIST for $\geq$ four weeks from the first demonstration of that rating, was significantly higher in the sorafenib group than in the placebo group ( $43 \%$ versus $32 \% ; P=0.002)$. Based on the SHARP data, sorafenib was approved by the European Medicines Agency in October 2007 and by the US Food and Drug Administration in November 2007 for the treatment of patients with unresectable HCC.

In order to achieve regulatory approval in China, Cheng et al conducted a Phase III randomized study comparing sorafenib with placebo in patients with advanced HCC in the Asia-Pacific region. ${ }^{37}$ This study had no predefined primary endpoint, and used the same eligibility criteria as the SHARP study. Two hundred and twenty-six patients in China, South Korea, and Taiwan were randomized on an intention-to-treat basis in a 2:1 ratio to sorafenib versus placebo. The number of patients needed for this study was calculated with a power of $83.9 \%$ to detect a $20 \%$ increase in overall survival in the sorafenib group. Overall survival, time to radiologic progression, time to symptomatic progression, disease-control rate, and safety were assessed. Patient characteristics and

Table 2 Summary of outcome in SHARP and Asia-Pacific studies

\begin{tabular}{|c|c|c|c|c|c|c|}
\hline \multirow[t]{2}{*}{ Efficacy parameter } & \multicolumn{3}{|l|}{ SHARP } & \multicolumn{3}{|c|}{ Asia-Pacific } \\
\hline & $\begin{array}{l}\text { Sorafenib } \\
(n=299)\end{array}$ & $\begin{array}{l}\text { Placebo } \\
(n=303)\end{array}$ & HR & $\begin{array}{l}\text { Sorafenib } \\
(n=I 50)\end{array}$ & $\begin{array}{l}\text { Placebo } \\
(n=76)\end{array}$ & HR \\
\hline OS (months) & 10.7 & 7.9 & $\begin{array}{l}0.69 \\
(P<0.001)\end{array}$ & 6.5 & 4.2 & $\begin{array}{l}0.68 \\
(P=0.0 \mid 4)\end{array}$ \\
\hline TTP (months) & 5.5 & 2.8 & $\begin{array}{l}0.58 \\
(P<0.00 I)\end{array}$ & 2.8 & 1.4 & $\begin{array}{l}0.57 \\
(P<0.00 \mathrm{I})\end{array}$ \\
\hline TTSP (months) & 4.1 & 4.9 & $\begin{array}{l}1.08 \\
(P=0.768)\end{array}$ & 3.5 & 3.4 & $\begin{array}{l}0.90 \\
(P=0.498)\end{array}$ \\
\hline DCR (\%) & 43 & 32 & $(P=0.002)$ & 35.3 & 15.8 & $(P=0.0019)$ \\
\hline
\end{tabular}

Abbreviations: HR, hazard ratio (sorafenib versus placebo); OS, overall survival; TTP, time to progression; TTSP, time to symptomatic progression; DCR, disease control rate. 
efficacy outcomes are summarized in Tables 1 and 2. Overall survival was 6.5 months with sorafenib versus 4.2 months in the placebo group, with an HR of $0.68(P=0.014)$. Additionally, there were significant differences in time to radiologic progression and disease-control rate between the sorafenib and placebo groups, but not in time to symptomatic progression.

The Asia-Pacific study has confirmed the results of the SHARP study, and the magnitude of benefits in overall survival and time to radiologic progression derived from sorafenib was almost the same between these two studies, ie, a $31 \%-32 \%$ of relative risk reduction in death, and a $42 \%-43 \%$ relative benefit of increase in time to progression. However, the absolute benefit of the increase in overall survival and time to radiologic progression derived from sorafenib was smaller in the Asia-Pacific study than in the SHARP study. Additionally, the median overall survival and time to radiologic progression were shorter in Asia-Pacific study. There were more patients with HBV and severe disease, such as macroscopic vascular invasion and/or extrahepatic spread in the Asia-Pacific study, which might have contributed to the shorter overall survival and time to radiologic progression than were seen in the SHARP trial.

Patients with HBV-related HCC were shown to have poorer overall survival than patients with $\mathrm{HCV}$-related HCC. ${ }^{38}$ HBV-related HCC was noted in $73 \%$ of patients in the AsiaPacific study versus $18 \%$ in the SHARP trial. A subset analysis of 165 patients with HBV-related HCC in the Asia-Pacific study indicated that sorafenib improved overall survival and time to radiologic progression independent of HBV status, and the safety profile of sorafenib in patients with HBV was comparable with that for the overall study patients. ${ }^{39}$ In the SHARP study, $30 \%$ of patients were infected with HCV, and $25 \%$ had alcoholic liver disease. In a subset analysis of 178 patients with HCV-related HCC in the SHARP trial, overall survival was 14.0 months in 93 patients receiving sorafenib versus 7.9 months in 85 patients receiving placebo. The disease-control rate was higher in the sorafenib group compared with placebo (44\% versus $31 \%$ ). The safety profile of sorafenib treatment in patients with $\mathrm{HCV}$-associated $\mathrm{HCC}$ was similar to that in all patients receiving sorafenib in this study. ${ }^{40}$ The subgroup analysis in patients with alcohol-related HCC in the SHARP study demonstrated a similar outcome and safety profile to the overall population. ${ }^{41}$ Taken together, there is a consistent clinical benefit from sorafenib in $\mathrm{HCC}$, irrespective of $\mathrm{HBV} / \mathrm{HCV}$ status or alcoholic liver disease.

Combined analysis of the SHARP and Asia-Pacific trials to examine the effects of macroscopic vascular invasion, extrahepatic spread, and Eastern Cooperative Oncology Group (ECOG) performance status on outcome has shown that sorafenib was effective and safe for the treatment of advanced HCC in patients globally, irrespective of baseline ECOG performance status and the presence or absence of macroscopic vascular invasion and/or extrahepatic spread. ${ }^{42}$ In the SHARP study, 38\% of patients had macroscopic vascular invasion and 52\% had extrahepatic spread versus 35\% with macroscopic vascular invasion and 68\% with extrahepatic spread in the Asia-Pacific study (Table 1). However, a significant difference in overall survival based on the presence or absence of macroscopic vascular invasion and/or extrahepatic spread was observed in both trials. For patients treated with sorafenib in the SHARP study, median overall survival was 14.5 months in patients without macroscopic vascular invasion or extrahepatic spread versus 8.9 months with macroscopic vascular invasion and/or extrahepatic spread. For patients treated with sorafenib in the Asia-Pacific trial, median overall survival was 14.3 and 5.6 months, respectively. Therefore, for advanced $\mathrm{HCC}$ patients with macroscopic vascular invasion and/or extrahepatic spread, there is a dire need for treatment options in addition to sorafenib to improve the outcome.

\section{Safety, tolerability, and patient-focused perspectives}

As summarized in Table 3, the common drug-related adverse events in patients receiving sorafenib were hand-foot skin reaction, diarrhea, alopecia, fatigue, skin rash, hypertension, nausea, and anorexia. Hand-foot skin reaction and diarrhea were the two most frequent severe (Grade 3 or 4 ) toxicities in patients receiving sorafenib, at about 5\%-10\%. For patients on sorafenib, 26\%-31\% required dose reduction, and $20 \%-38 \%$ of patients terminated treatment due to toxicities. There seemed to be less treatment discontinuation in the Asia-Pacific study regardless of whether patients were on sorafenib or placebo than in the SHARP study.

There might be ethnic differences in hand-foot skin reaction, which occurred more frequently in Asian patients. Hand-foot skin reaction is manifested by formation of thick hyperkeratotic lesions affecting pressure points and flexure areas. ${ }^{43}$ These lesions usually develop after 2-4 weeks on sorafenib treatment and are often painful, which affects patient quality of life. ${ }^{44} \mathrm{~A}$ mild hand-foot skin reaction can be treated with lanolin-based or urea-based lotions. Protective measures, such as wearing gloves and socks, are recommended to provide pain relief and prevent skin breakdown. Dose reduction or interruption of sorafenib is frequently needed in patients 
Table 3 Incidence of drug-related adverse effects

\begin{tabular}{|c|c|c|c|c|c|c|c|c|}
\hline \multirow[t]{3}{*}{ Toxicities } & \multicolumn{4}{|c|}{ SHARP } & \multicolumn{4}{|c|}{ Asia-Pacific } \\
\hline & \multicolumn{2}{|c|}{ Sorafenib } & \multicolumn{2}{|c|}{ Placebo } & \multicolumn{2}{|c|}{ Sorafenib } & \multicolumn{2}{|c|}{ Placebo } \\
\hline & All & Grade 3/4 & All & Grade 3/4 & All & Grade 3/4 & All & Grade $3 / 4$ \\
\hline Hand-foot skin reaction & $21^{a}$ & 8 & 3 & $<1$ & 45 & 11 & 3 & 0 \\
\hline Diarrhea & 39 & 8 & 11 & 2 & 26 & 6 & 5 & 0 \\
\hline Alopecia & 14 & 0 & 2 & 0 & 25 & 0 & I & 0 \\
\hline Fatigue & 22 & 4 & 16 & 4 & 20 & 3 & 8 & I \\
\hline Rash/desquamation & 16 & I & 11 & 0 & 20 & I & 7 & 0 \\
\hline Hypertension & 5 & 2 & 2 & I & 19 & 2 & I & 0 \\
\hline Nausea/vomiting & 16 & 2 & 11 & 2 & 11 & I & 11 & I \\
\hline Anorexia & 14 & $<1$ & 3 & I & 13 & 0 & 3 & 0 \\
\hline Dose reduction ${ }^{\mathrm{b}}$ & 26 & & 7 & & 31 & & 3 & \\
\hline Drug discontinuation ${ }^{\mathrm{b}}$ & 38 & & 37 & & 20 & & 13 & \\
\hline
\end{tabular}

Notes: ${ }^{a}$ Variables are expressed as percentages; 'bue to adverse events.

with moderate to severe hand-foot skin reaction. Sorafenib can be resumed at a lower dose, with gradual escalation when hand-foot skin reaction is resolved.

Adverse cardiovascular toxicities, including myocardial infarction, hypertension, congestive heart failure, and arrhythmia, are frequently associated with antiangiogenic inhibitors, and are regarded as a class effect. ${ }^{45}$ Hypertension is the most frequent event, and most of the time is clinically manageable. The incidence of hypertension was $5 \%$ in the SHARP study and $19 \%$ in the Asia-Pacific study, with a $2 \%$ incidence of Grade 3 or 4 hypertension noted in sorafenibtreated patients in each of the studies. The incidence of severe cardiac ischemia or infarction in sorafenib-treated patients in the SHARP study was 3\% versus $1 \%$ in patients taking placebo. ${ }^{36}$ Sorafenib was associated with hypophosphatemia in $35 \%$ of sorafenib-treated patients compared with $11 \%$ taking placebo in the SHARP study. Grade 3 or 4 hypophosphatemia, which can lead to muscle weakness, altered mental status, and cardiac events, was seen in $11 \%$ of patients taking sorafenib. ${ }^{32}$ Therefore, sorafenib is not recommended in patients with a recent history of cardiac disease. All patients taking sorafenib should be monitored frequently for symptoms of cardiac disease and have blood levels monitored regularly for hypophosphatemia.

Hypothyroidism is a common side effect of sorafenib, and the incidence can be as high as $18 \%{ }^{46}$ Sorafenib can enhance T4 and T3 metabolism, which is probably caused by increased Type 3 deiodination. ${ }^{47}$ Clinical hypothyroidism occurs about one to seven months after the initiation of sorafenib. ${ }^{48}$ Therefore, it is important to monitor thyroid function periodically in patients receiving sorafenib. In the SHARP study, elevated lipase occurred in $40 \%$ of patients receiving sorafenib versus $37 \%$ for patients on placebo. However, Grade 3 or 4 lipase elevation was seen in $9 \%$ of patients receiving sorafenib. ${ }^{32}$ There have been cases reported of acute pancreatitis in patients receiving sorafenib treatment for HCC or renal cell carcinoma, and pancreatitis usually improves after stopping sorafenib. ${ }^{49-51}$

There are limited data on the use of sorafenib in patients with Child-Pugh B or Child-Pugh C hepatic impairment. Yau et al reported a study involving 15 patients with Child-Pugh B/C cirrhosis receiving sorafenib for HCC. Thirteen patients discontinued treatment prematurely due to treatment toxicities. ${ }^{52}$ In a Phase II study of sorafenib in HCC, including 98 Child-Pugh A and 38 Child-Pugh B patients, there was no significant difference in sorafenib pharmacokinetics between patients with Child-Pugh A and Child-Pugh B impairment. ${ }^{53}$ However, Child-Pugh B patients had a higher rate of hyperbilirubinemia, encephalopathy, and worsening ascites, and Child-Pugh B patients did worse, with overall survival of 14 weeks versus 41 weeks for patients with Child-Pugh A. Therefore, it is recommended to reduce the starting dose, perhaps by $50 \%$, with vigilant monitoring of liver function, if sorafenib is to be used in patients with Child-Pugh B liver cirrhosis. ${ }^{35,54}$ For HCC patients with Child-Pugh $\mathrm{C}$, because of their severe underlying disease with limited life expectancy of usually less than three months, sorafenib treatment is not recommended. ${ }^{55,56}$

In both the SHARP and Asian-Pacific studies, the Functional Assessment of Cancer Therapy Hepatobiliary Symptom Index 8 (FHSI-8) was used to assess time to symptomatic progression. FHSI- 8 is a patient-oriented outcome instrument designed to assess symptoms and quality of life in hepatobiliary cancer patients in general. ${ }^{57}$ FHSI- 8 analyses in both studies failed to identify any significant difference between the sorafenib and placebo groups. The quality of life of these patients might have been affected by other concurrent diseases, such as liver cirrhosis. Additionally, FHSI-8 might not have 

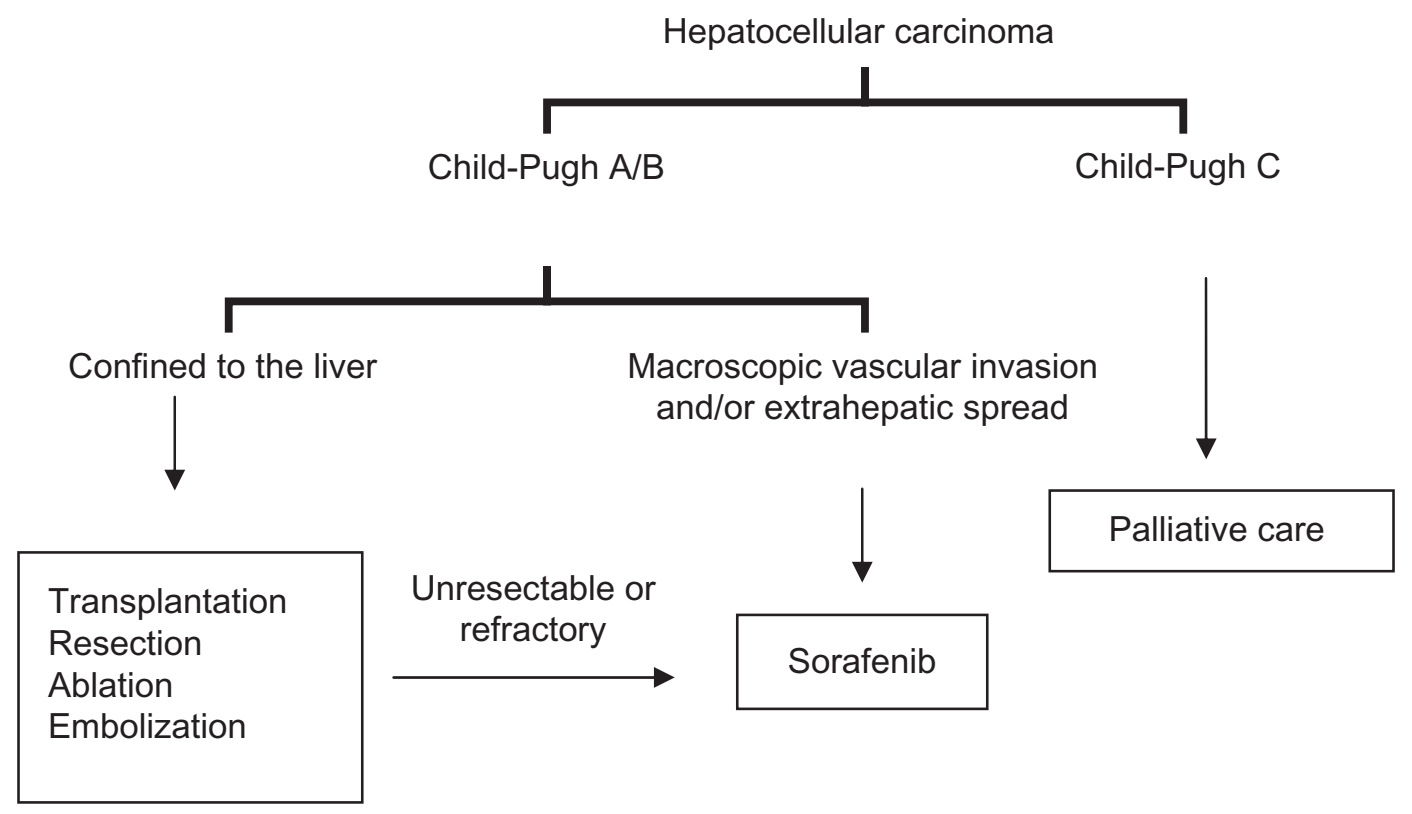

Figure I A simplified treatment schema for hepatocellular carcinoma.

been sensitive enough to determine small but significant differences in quality of life between these two groups.

\section{Conclusion}

Sorafenib is the first agent that has resulted in a survival benefit in HCC, and has validated the role of targeted therapy in this disease. Its role in the management of advanced unresectable cancer is now well proven in all subpopulations of HCC having adequate hepatic reserve, and has been incorporated in the consensus-based treatment algorithm globally (see Figure 1 for a simplified schema). ${ }^{58-60}$ The role of sorafenib in HCC confined to the liver and as part of multimodality HCC treatment is currently under investigation. Ongoing studies will incorporate sorafenib with other treatment modalities, including transarterial chemoembolization, cytotoxic chemotherapy, surgery, and transplantation, in HCC patients. ${ }^{61}$ Further studies are needed to define its dosage and role in patients with ChildPugh B and Child-Pugh C liver cirrhosis. Identification of a predictive biomarker signature will help to define the ideal patient population for sorafenib treatment in HCC.

\section{Disclosure}

The authors report no conflicts of interest in this work.

\section{References}

1. Bosch FX, Ribes J, Diaz M, Cleries R. Primary liver cancer: Worldwide incidence and trends. Gastroenterology. 2004;127(5 Suppl 1):S5-S16.

2. Benvegnu L, Gios M, Boccato S, Alberti A. Natural history of compensated viral cirrhosis: A prospective study on the incidence and hierarchy of major complications. Gut. 2004;53(5):744-749.
3. El-Serag HB, Davila JA, Petersen NJ, McGlynn KA. The continuing increase in the incidence of hepatocellular carcinoma in the United States: An update. Ann Intern Med. 2003;139(10):817-823.

4. Marrero JA, Welling T. Modern diagnosis and management of hepatocellular carcinoma. Clin Liver Dis. 2009;13(2):233-247.

5. Chang MH, Chen CJ, Lai MS, et al. Universal hepatitis B vaccination in Taiwan and the incidence of hepatocellular carcinoma in children. Taiwan Childhood Hepatoma Study Group. $N$ Engl J Med. 1997;336(26):1855-1859.

6. Fattovich G, Stroffolini T, Zagni I, Donato F. Hepatocellular carcinoma in cirrhosis: Incidence and risk factors. Gastroenterology. 2004;127 (5 Suppl 1):S35-50.

7. Llovet JM, Schwartz M, Mazzaferro V. Resection and liver transplantation for hepatocellular carcinoma. Semin Liver Dis. 2005;25(2): $181-200$.

8. Mazzaferro V, Regalia E, Doci R, et al. Liver transplantation for the treatment of small hepatocellular carcinomas in patients with cirrhosis. N Engl J Med. 1996;334(11):693-699.

9. Majno P, Giostra E, Morel P, Hadengue A, Mentha G. Management of hepatocellular carcinoma in the waiting list before liver transplantation. J Hepatol. 2005;42 Supp1 1:S134-S143.

10. Livraghi T, Goldberg SN, Lazzaroni S, Meloni F, Solbiati L, Gazelle GS. Small hepatocellular carcinoma: Treatment with radio-frequency ablation versus ethanol injection. Radiology. 1999;210(3):655-661.

11. Lencioni RA, Allgaier HP, Cioni D, et al. Small hepatocellular carcinoma in cirrhosis: Randomized comparison of radio-frequency thermal ablation versus percutaneous ethanol injection. Radiology. 2003; 228(1):235-240

12. Lin SM, Lin CJ, Lin CC, Hsu CW, Chen YC. Radiofrequency ablation improves prognosis compared with ethanol injection for hepatocellular carcinoma $\leq 4 \mathrm{~cm}$. Gastroenterology. 2004;127(6):1714-1723.

13. Shiina S, Teratani T, Obi S, et al. A randomized controlled trial of radiofrequency ablation with ethanol injection for small hepatocellular carcinoma. Gastroenterology. 2005;129(1):122-130.

14. Llovet JM, Bruix J. Systematic review of randomized trials for unresectable hepatocellular carcinoma: Chemoembolization improves survival. Hepatology. 2003;37(2):429-442.

15. Kulik LM, Carr BI, Mulcahy MF, et al. Safety and efficacy of $90 \mathrm{Y}$ radiotherapy for hepatocellular carcinoma with and without portal vein thrombosis. Hepatology. 2008;47(1):71-81. 
16. Carr BI, Kondragunta V, Buch SC, Branch RA. Therapeutic equivalence in survival for hepatic arterial chemoembolization and yttrium 90 microsphere treatments in unresectable hepatocellular carcinoma. Cancer. 2010;116(5):1305-1314.

17. Thomas MB, O'Beirne JP, Furuse J, Chan AT, Abou-Alfa G, Johnson P. Systemic therapy for hepatocellular carcinoma: Cytotoxic chemotherapy, targeted therapy and immunotherapy. Ann Surg Oncol. 2008; 15(4):1008-1014.

18. Finn RS. Development of molecularly targeted therapies in hepatocellular carcinoma: Where do we go now? Clin Cancer Res. 2010;16(2): 390-397.

19. Adnane L, Trail PA, Taylor I, et al. Sorafenib (BAY 43-9006, Nexavar), a dual-action inhibitor that targets RAF/MEK/ERK pathway in tumor cells and tyrosine kinases VEGFR/PDGFR in tumor vasculature. Methods Enzymol. 2006;407:597-612.

20. Strumberg D. Preclinical and clinical development of the oral multikinase inhibitor sorafenib in cancer treatment. Drugs Today (Barc). 2005;41(12):773-784.

21. Gollob JA, Wilhelm S, Carter C, Kelley SL. Role of Raf kinase in cancer: Therapeutic potential of targeting the Raf/MEK/ERK signal transduction pathway. Semin Oncol. 2006;33(4):392-406.

22. Liu L, Cao Y, Chen C, et al. Sorafenib blocks the RAF/MEK/ERK pathway, inhibits tumor angiogenesis, and induces tumor cell apoptosis in hepatocellular carcinoma model PLC/PRF/5. Cancer Res. 2006; 66(24):11851-11858.

23. Strumberg D, Richly H, Hilger RA, et al. Phase I clinical and pharmacokinetic study of the novel Raf kinase and vascular endothelial growth factor receptor inhibitor BAY 43-9006 in patients with advanced refractory solid tumors. J Clin Oncol. 2005;23(5):965-972.

24. Abou-Alfa GK, Schwartz L, Ricci S, et al. Phase II study of sorafenib in patients with advanced hepatocellular carcinoma. J Clin Oncol. 2006; 24(26):4293-4300.

25. Llovet JM, Peña C, Shan M, Lathia C, Bruix J. Biomarkers predicting outcome of patients with advanced hepatocellular carcinoma (HCC) randomized in the Phase III SHARP trial. Hepatology. 2008; 48(S1):372A.

26. Sorafenib: Summary of product characteristics. Available from: http://www.emea.europa.eu/humandocs/PDFs/EPAR/nexavar/emeacombined-h690en.pdf. Accessed 2010 Oct 12.

27. Lathia C, Lettieri J, Cihon F, Gallentine M, Radtke M, Sundaresan P. Lack of effect of ketoconazole-mediated CYP3A inhibition on sorafenib clinical pharmacokinetics. Cancer Chemother Pharmacol. 2006;57(5):685-692.

28. Strumberg D, Clark JW, Awada A, et al. Safety, pharmacokinetics, and preliminary antitumor activity of sorafenib: A review of four phase I trials in patients with advanced refractory solid tumors. Oncologist. 2007;12(4):426-437.

29. Awada A, Hendlisz A, Gil T, et al. Phase I safety and pharmacokinetics of BAY 43-9006 administered for 21 days on/7 days off in patients with advanced, refractory solid tumours. $\mathrm{Br} J$ Cancer. 2005;92(10):1855-1861.

30. Sorabenib U.S. prescribing information. Available from: http://www. nexavar.com/html/download/Nexavar_PI.pdf. Accessed 2010 Oct 12.

31. Moretti LV, Montalvo RO. Elevated International Normalized Ratio associated with concurrent use of sorafenib and warfarin. Am J Health Syst Pharm. 2009;66(23):2123-2125.

32. Kane RC, Farrell AT, Madabushi R, et al. Sorafenib for the treatment of unresectable hepatocellular carcinoma. Oncologist. 2009;14(1):95-100.

33. Pugh RN, Murray-Lyon IM, Dawson JL, Pietroni MC, Williams R. Transection of the oesophagus for bleeding oesophageal varices. $\mathrm{Br} J$ Surg. 1973;60(8):646-649.

34. Furuse J, Ishii H, Nakachi K, Suzuki E, Shimizu S, Nakajima K. Phase I study of sorafenib in Japanese patients with hepatocellular carcinoma. Cancer Sci. 2008;99(1):159-165.

35. Miller AA, Murry DJ, Owzar K, et al. Phase I and pharmacokinetic study of sorafenib in patients with hepatic or renal dysfunction: CALGB 60301. J Clin Oncol. 2009;27(11):1800-1805.
36. Llovet JM, Ricci S, Mazzaferro V, et al. Sorafenib in advanced hepatocellular carcinoma. $N$ Engl J Med. 2008;359(4):378-390.

37. Cheng A-L, Kang Y-K, Chen Z, et al. Efficacy and safety of sorafenib in patients in the Asia-Pacific region with advanced hepatocellular carcinoma: A phase III randomised, double-blind, placebo-controlled trial. Lancet Oncol. 2009;10(1):25-34.

38. Cantarini MC, Trevisani F, Morselli-Labate AM, et al. Effect of the etiology of viral cirrhosis on the survival of patients with hepatocellular carcinoma. Am J Gastroenterol. 2006;101(1):91-98.

39. Guan Z, Kang Y, Chen Z, et al. Sorafenib is effective in hepatitis B-positive patients with hepatocellular carcinoma: Subgroup analysis of a randomized, double-blind, phase III trial performed in the AsiaPacific region. Ann Oncol. 2008;19 Suppl 8:viii166-viii186.

40. Bolondi L, Caspary W, Bennouna J, et al. Clinical benefit of sorafenib in hepatitis $C$ patients with hepatocellular carcinoma: Subgroup analysis of the SHARP trial. Paper presented at Gastrointestinal Cancers Symposium, Orlando, FL, 2008 Jan 25-27.

41. Craxi A, Porta C, Sangiovanni A, et al. Efficacy and safety of sorafenib in patients with alcohol-related hepatocellular carcinoma: A sub-analysis from the SHARP trial. J Clin Oncol. 2008;26 Suppl 15:15591.

42. Bruix J, Cheng A, Kang Y, et al. Effect of macroscopic vascular invasion (MVI), extrahepatic spread (EHS), and ECOG performance status (ECOG PS) on outcome in patients with advanced hepatocellular carcinoma (HCC) treated with sorafenib: Analysis of two phase III, randomized, double-blind trials. J Clin Oncol. 2009;27 Suppl 15:4580.

43. Lacouture ME, Wu S, Robert C, et al. Evolving strategies for the management of hand-foot skin reaction associated with the multitargeted kinase inhibitors sorafenib and sunitinib. Oncologist. 2008;13(9): 1001-1011.

44. Chu D, Lacouture ME, Fillos T, Wu S. Risk of hand-foot skin reaction with sorafenib: A systematic review and meta-analysis. Acta Oncol. 2008;47(2):176-186.

45. Cheng H, Force T. Molecular mechanisms of cardiovascular toxicity of targeted cancer therapeutics. Circ Res. 2010;106(1):21-34.

46. Torino F, Corsello SM, Longo R, Barnabei A, Gasparini G. Hypothyroidism related to tyrosine kinase inhibitors: An emerging toxic effect of targeted therapy. Nat Rev Clin Oncol. 2009;6(4):219-228.

47. Abdulrahman RM, Verloop H, Hoftijzer H, et al. Sorafenib-induced hypothyroidism is associated with increased Type 3 deiodination. J Clin Endocrinol Metab. 2010;95(8):3758-3762.

48. Walko CM, Aubert RE, La-Beck NM, et al. Pharmacoepidemiology of clinically relevant hypothyroidism and hypertension from sunitinib and sorafenib. J Clin Oncol. 2010;28 Suppl 15:9149.

49. Saadati H, Saif MW. Sorafenib-induced acute pancreatitis. JOP. 2010;11(3):283-284.

50. Li M, Srinivas S. Acute pancreatitis associated with sorafenib. South Med J. 2007;100(9):909-911.

51. Amar S, Wu KJ, Tan WW. Sorafenib-induced pancreatitis. Mayo Clin Proc. 2007;82(4):521.

52. Yau T, Chan P, Ng K, Chok K, Fan ST, Poon R. Efficacy and tolerability of single agent sorafenib in poor risk advanced hepatocellular carcinoma patients. J Clin Oncol. 2008;26 Suppl 15:15513.

53. Abou-Alfa GK, Amadori D, Santoro A, et al. Is sorafenib (S) safe and effective in patients (pts) with hepatocellular carcinoma (HCC) and Child-Pugh B (CPB) cirrhosis? J Clin Oncol. 2008;26 Suppl 15:4518.

54. Abou-Alfa GK. Selection of patients with hepatocellular carcinoma for sorafenib. J Natl Compr Canc Netw. 2009;7(4):397-403.

55. Pinter M, Sieghart W, Graziadei I, et al. Sorafenib in unresectable hepatocellular carcinoma from mild to advanced stage liver cirrhosis. Oncologist. 2009;14(1):70-76.

56. Worns MA, Weinmann A, Pfingst K, et al. Safety and efficacy of sorafenib in patients with advanced hepatocellular carcinoma in consideration of concomitant stage of liver cirrhosis. J Clin Gastroenterol. 2009;43(5):489-495.

57. Sebastian E, Arnold JS, Susan A, Susan E, Kelly MM, Robert CGM. Assessment of symptom experience in patients undergoing hepatic resection or ablation. Cancer. 2006;107(11):2715-2722. 
58. Benson AB 3rd, Abrams TA, Ben-Josef E, et al. NCCN clinical practice guidelines in oncology: Hepatobiliary cancers. J Natl Compr Canc Netw. 2009;7(4):350-391.

59. Kudo M, Ueshima K. Positioning of a molecular-targeted agent, sorafenib, in the treatment algorithm for hepatocellular carcinoma and implication of many complete remission cases in Japan. Oncology. 2010; 78 Suppl 1:154-166.
60. Chen PJ, Furuse J, Han KH, et al. Issues and controversies of hepatocellular carcinoma-targeted therapy clinical trials in Asia: Experts' opinion. Liver Int. 2010 Jun 15. [Epub ahead of print].

61. Zhu AX. Systemic treatment of hepatocellular carcinoma: Dawn of a new era? Ann Surg Oncol. 2010;17(5):1247-1256.
Hepatic Medicine: Evidence and Research

\section{Publish your work in this journal}

Hepatic Medicine: Evidence and Research is an international, peerreviewed, open access journal covering all aspects of adult and pediatric hepatology in the clinic and laboratory including the following topics: Pathology, pathophysiology of hepatic disease; Investigation and treatment of hepatic disease; Pharmacology of drugs used for

\section{Dovepress}

the treatment of hepatic disease. Issues of patient safety and quality of care will also be considered. The manuscript management system is completely online and includes a very quick and fair peer-review system, which is all easy to use. Visit http:/www.dovepress.com/ testimonials.php to read real quotes from published authors.

Submit your manuscript here: http://www.dovepress.com/hepatic-medicine-evidence-and-research-journal 